\title{
Editorial
}

\section{Austausch pädagogischer Ideen auf dem Papier. Pädagogische Zeitschriften und transnationaler Wissenstransfer (1850-2000)}

\section{Béatrice Haenggeli-Jenni, Alexandre Fontaine und Patrick Bühler}

Spätestens seit der Mitte des 18. Jahrhunderts trägt die intensive Nutzung von Zeitschriften in der Pädagogik zur Standardisierung schulischer Praktiken bei. Pädagoginnen und Pädagogen, Begriffe und Wissen zirkulieren; pädagogische Konzepte werden im Hinblick auf die lokalen Bedürfnisse diskutiert und ausgetauscht. In den Schulen Europas kommt es zu einer Angleichung, so werden nationale Schulsysteme etwa an Weltausstellungen (Schröder-Gudehus \& Rasmussen, 1992; Lawn, 2009; Dittrich, 2010) oder eben in der wissenschaftlichen Literatur untereinander verglichen. Die pädagogische Presse ist somit Teil eines umfassenden Vergleichsprozesses, der international bei der Entstehung von Nationalstaaten beobachtet werden kann. In der zweiten Hälfte des 19. Jahrhunderts ist die rege Nutzung von Zeitschriften aber auch Teil eines Entwicklungsprozesses, in dem Periodika für die Wissenschaft zunehmend an Bedeutung gewinnen. Zeitschriften werden zu einem Medium des Austauschs, das Julliard (2007) ein «Buch mit mehreren Stimmen», eine «Denk- und Kommunikationskooperative» nennt, in der jeder Autor nicht so sehr seine eigenen Ideen vorstellt, sondern vor allem die Zirkulation von Wissen vorantreibt. So seien Zeitschriften Julliard zufolge eines der Merkmale der Wissenschaft, in der sich Gedanken in «ständiger Konfrontation» befänden: in «Konfrontation eines Menschen mit anderen Menschen, eines Denkers mit seiner Zeit» (Julliard, 1987, S. 3). Das ist einer der Gründe, weshalb Periodika von Soziologen und Historikern, die sich mit der Entwicklung wissenschaftlicher Disziplinen befassen, gerne erforscht werden. Schriewer (1998) betont beispielsweise, dass Zeitschriften «die Messinstrumente par excellence sind, durch die der fachliche Kommunikationsprozess weiterentwickelt, gestaltet, konstituiert» werde (S. 59). Auch Hofstetter und Schneuwly (2004) vergleichen Periodika mit «Arenen, in denen die Forschungsergebnisse erstmals dargelegt und debattiert» würden. Dadurch trügen Zeitschriften zur Schaffung einer «Forschergemeinschaft» bei, die gemeinsame Fragestellungen in einer gemeinsamen Sprache untersuche (S. 575). In der Pädagogik gibt es natürlich nicht nur wissenschaftliche Zeitschriften, sondern auch Lehrer-, Gewerkschafts- oder Vereinszeitschriften. Aber auch diesen kommt, wie 
Caspard (2006) zeigt, die doppelte Aufgabe zu, zu informieren und zur Debatte zu stellen, indem zum einen "ganz unterschiedliche bildungspolitische Umsetzungen» erfasst würden und zum anderen eine "wahre Anthologie der pädagogischen Reflexion» dargeboten werde, «die nicht nur die grossen, sondern auch die kleineren Pädagogen, die viel zahlreicher sind, zu Wort kommen» lasse (S. 565). Zeitschriften sind somit ein ausgezeichnetes Mittel, um die Entwicklung der Pädagogik zu studieren. Denn Periodika erlauben es, Prozesse der Rezeption, der Readaption und der Resemantisierung (Espagne, 1999; Fontaine, 2013) $\mathrm{zu}$ analysieren. So werden in Zeitschriften ganz unterschiedliche Themen wie pädagogische Methoden, Unterrichtsformen, die Organisation von Bildungssystemen oder die philosophische Bedeutung der Erziehung behandelt. Solche Themen werden häufig schnell über die Landesgrenzen hinweg diskutiert und so lässt sich der Austausch von Schulkonzepten und -modellen zwischen Regionen untersuchen (Steiner-Khamsi, 2004; Matasci, 2012).

Die vorliegende Ausgabe der Schweizerischen Zeitschrift für Bildungswissenschaften vereinigt daher Beiträge, die pädagogische Zeitschriften als Teil eines kulturellen Austauschs analysieren. In dieser Ausgabe werden unterschiedliche methodische Ansätze verfolgt, die jedoch alle versuchen, die Modalitäten solcher Transfers auf dem Papier zu erfassen und die Bedeutung der pädagogischen Presse bei der disziplinären Entwicklung zu studieren. Es gilt zu überprüfen, ob Zeitschriften tatsächlich privilegierte Orte des Austauschs sind, ob Periodika die Zirkulation pädagogischen Wissens befördern - lokal, regional, national, transnational. So soll durch die Analyse von Zeitschriften erforscht werden, welche Inhalte übernommen, über welche Kanäle sie verbreitet und wie sie rezipiert werden. Indem ein besonderes Augenmerk auf die redaktionellen Eigenheiten der Zeitschriften (Redaktionsausschuss, Erscheinungshäufigkeit, Art der Verbreitung, Leserschaft, Wettbewerb zwischen den Zeitschriften etc.) sowie auf die Präsentation des Inhalts (Titel der Artikel, Chroniken, wiederkehrende Rubriken usw.) gerichtet wird, kann untersucht werden, wie pädagogisches Wissen verändert und verbreitet wird. Die pädagogische Presse ist ein wichtiges Hilfsmittel, um den transnationalen Aufbau der Pädagogik zu verstehen und um zu sehen, wie dabei «fremdes» Wissen einbezogen wird.

Wie Alexandre Fontaine zeigt, ist die französische Schweiz rege an diesem internationalen Austausch beteiligt. In seinem Beitrag untersucht Fontaine die Dynamik des Transfers, der zwischen 1865 und 1890 in L'Éducateur auf interregionaler und transnationaler Ebene stattfindet. Fontaine zeigt u. a. die zwiespältige Beziehung zur Deutschschweiz und besonders zum Deutschschweizer Lehrerverband auf. Während sich das Verhältnis zwischen den beiden Lagern mit der Zeit zumindest rhetorisch abzukühlen scheint, erforscht tatsächlich jeder die vom anderen entwickelten Neuheiten, um sie anschliessend in der eigenen Zeitschrift zu veröffentlichen. Der länderübergreifende Austausch scheint trotz allem fruchtbarer zu sein, denn zahlreiche Artikel des L'Éducateur werden in ausländischen Zeitschriften übernommen, während das Organ der Société des 
instituteurs romands ebenfalls Beiträge aus dem Ausland (hauptsächlich aus Europa) publiziert. Besondere Beachtung wird nicht nur dem Direktor der Zeitschrift Alexandre Daguet geschenkt, sondern auch anderen wichtigen Akteuren des Austauschs. Zu ihnen gehört z. B. Caroline Progler, die in der Romandie via $L$ 'Éducateur die Pädagogik Friedrich Fröbels verbreitet.

Monique Mombert zeichnet in ihrem Beitrag nach, wie ein deutsch-französischer Konkurrenzkampf die Gründung des französischen Bulletin de la Société des Professeurs de Langues (1903) vorantreibt, das 1907 zur Zeitschrift Les Langues modernes wird. Deren Herausgeber Charles Sigwalt ist überzeugt, dass die pädagogische Presse bei der Schaffung eines Fachverbands für Fremdsprachenlehrer eine zentrale Rolle spiele. Er betont dabei das Fehlen von Publikationen, um die Bedeutung seiner eigenen Zeitschrift zu erhöhen. Mombert zeigt nun, dass es sehr wohl mehrere Zeitschriften gibt, die mit Les Langues modernes in Konkurrenz stehen. Dazu gehören u. a. Wilhelm Viëtors Die Neueren Sprachen (1893) oder Auguste Wolfromms Revue de l'Enseignement des Langues vivantes (1884). Indem Mombert die Zirkulation von Wissen zwischen diesen Zeitschriften untersucht, kann sie zeigen, dass alle drei Zeitschriften eine Schlüsselrolle bei der Professionalisierung der Redaktoren gespielt und dabei sich wissenschaftlich einander angenähert haben.

Patrick Bühler untersucht die unterschiedliche Rezeption der psychoanalytischen Pädagogik in Europa. Während die Anwendung der Psychoanalyse in der Pädagogik in Deutschland meist auf Ablehnung stösst, blüht sie am Institut Jean-Jacques Rousseau in Genf ebenso wie auf der anderen Seite der Schweizer Sprachgrenze. So geben zwei Pioniere der psychoanalytischen Pädagogik, der Berner Seminardirektor Ernst Schneider und der Zürcher Pfarrer Oskar Pfister, die 1907 gegründeten reformpädagogischen Berner Seminarblätter (ab 1916 Die Schulreform) heraus. Darin propagiert Pfister etwa seine "Pädanalyse», an der u. a. Freud Kritik übt. Bühler unterstreicht die zwiespältige Rolle, welche die Seminarblätter bei der Verbreitung einer psychoanalytischen Pädagogik spielen. Das reformpädagogische Interesse fällt nämlich nur gering aus und die Rezeption der Psychoanalyse erweist sich als ausgesprochen selektiv.

Frédéric Mole analysiert die Zirkulation pädagogischer Konzepte in zwei französischen Gewerkschaftszeitschriften: L'École Emancipée, Organ der (revolutionären) Lehrergewerkschaft Fédération Unitaire de l'Enseignement, und L'École Libératrice, Zeitschrift des (reformistischen) Lehrervereins Syndicat National des Instituteurs. Mole zeigt, wie die von der Ligue internationale pour l'Éducation nouvelle geförderten Methoden von den Redaktoren aufgenommen und diskutiert werden. So hebt der Autor die Rolle der Zeitungen als «Relais» hervor, die Wissen weitergeben, indem sie es ans Zielpublikum anpassen. Eine der Personen, die bei diesem Austausch ein doppeltes Spiel spielt, ist Célestin Freinet. Er ist Redaktor beider Zeitschriften und bewegt sich zwischen beiden Diskursen hin und her. Auf der einen Seite fördert er die neuartigen Methoden, um die Position der revolutionären Gewerkschafter weiterzuentwickeln, auf 
der anderen Seite kritisiert er in der reformistischen Zeitschrift eben diese Methoden. Freinet nutzt seine Stellung in den beiden Netzwerken aus, um seine manchmal paradoxe Einstellung vom einen Netzwerk ins andere weiterzugeben. Je nach verlegerischem Kontext finden somit ein Transfer, eine Anpassung und eine Neuausrichtung der pädagogischen Begriffe statt.

Der Artikel von Monika Knaupp, Sarah Schaufler, Susann Hofbauer und Edwin Keiner liefert schliesslich eine vergleichende Analyse mehrerer Zeitschriften aus der Erziehungswissenschaft und aus der pädagogischen Psychologie. Es werden Zeitschriften aus drei Ländern (Italien, Deutschland, England) verglichen und nach sozialen und methodischen Aspekten untersucht. Für die Erziehungswissenschaft zeigt sich, dass sie stark von nationalen Sprachund Kulturtraditionen abhängig ist und von den Gegebenheiten vor Ort beeinflusst wird. Wegen dieser Durchlässigkeit wird sie als «fractured-porous discipline» bezeichnet. Die pädagogische Psychologie hingegen erweist sich als eine eigenständigere, stärker abgeschlossene Disziplin mit einer strengen Methodik, die von der wissenschaftlichen Gemeinschaft, in der sich die Disziplin entwickelt, validiert wird. Es handelt sich somit um eine deutlicher transnationale Disziplin, deren Wissen innerhalb der Fachgemeinschaft zirkuliert und weniger von nationalen Unterschieden beeinflusst wird - Eigenschaften, welche sich als eine «unified-insular discipline» umschreiben lassen.

Die fünf Artikel in diesem Themenheft unterstreichen die Bedeutung von pädagogischen Zeitschriften als «Medium» des Wissensaustauschs. Solche Transfers tragen noch immer zur Entwicklung der Disziplin und zur Schaffung einer gemeinsamen Basis bei. Die Beiträge der vorliegenden Ausgabe gehen dem besonderen Charakter der pädagogischen Presse nach, die sich an der Schnittstelle von Wissenschaft und Beruf befindet. Empirisches, theoretisches, pädagogisches, psychologisches und philosophisches Wissen wird ausgetauscht, das sich in einem ständigen Hin und Her zwischen Beruf und Forschung entwickelt. Dieses Wissen wird in unterschiedlichen Kontexten absorbiert, interpretiert und resemantisiert, durch den Kontakt mit anderem Wissen erhält es so neue Facetten.

\section{Literatur}

Caspard, P. (2006). La presse d'éducation et d'enseignement. In T. Charmasson (Hrsg.), L'histoire de l'enseignement, XIX'-XXe siècles. Guide du chercheur (S. 569-579). Paris / Lyon: CTHS / INRP.

Dittrich, K. (2010). Experts going transnational: education at world exhibitions during the second half of the nineteenth century. University of Portsmouth, Dissertation.

Espagne, M. (1999). Les transferts culturels franco-allemands. Paris: PUF.

Fontaine, A. (2013). Transferts culturels et déclinaisons de la pédagogie européenne. Le cas francoromand au travers de l'itinéraire d'Alexandre Daguet (1816-1894). Université de Fribourg et de Paris 8, Dissertation.

Hofstetter, R. \& Schneuwly, B. (Hrsg.). (2004). The role of congresses and institutes in the emergence of the educational sciences. Paedagogica Historica (Special issue), 40 (5 \& 6). 
Julliard, J. (1987). Le monde des revues au début du siècle. Introduction. Revue Mil Neuf Cent, Numéro spécial: Les revues dans la vie intellectuelle (1885-1914), 5, 3-10.

Julliard, J. (2007). Avant-propos. Comment on se dispute. Les formes de la controverse. Revue Mil neuf cent, 25, 3-5.

Lawn, M. (2009). Modelling the Future. Exhibitions and the Materiality of Education. London: Symposium Books.

Matasci, D. (2012). L'école républicaine et l'étranger. Acteurs et espaces de l'internationalisation de la «réforme scolaire» en France (1870-première moitié du XXe siècle). Université de Genève \& EHESS, Dissertation.

Schriewer, J. (1998). Études pluridisciplinaires et réflexions philosophico-herméneutiques: la structuration du discours pédagogique en France et en Allemagne. In P. Drewek \& C. Lüth (Hrsg.), Histoire des sciences de liéducation (S. 57-84). Gent: CSHP.

Schröder-Gudehus, B. \& Rasmussen, A. (1992). Les fastes du progrès; le guide des expositions universelles, 1851-1992. Paris: Flammarion.

Steiner-Khamsi, G. (2004). The global politics of educational borrowing and lending. New York: Teachers College Press. 
